\title{
Molecular excitation in the Eagle nebula's fingers
}

\author{
F. Schuller ${ }^{1}$, S. Leurini ${ }^{1}$, C. Hieret $^{1}$, K. M. Menten ${ }^{1}$, S. D. Philipp ${ }^{1}$, R. Güsten ${ }^{1}$, P. Schilke ${ }^{1}$, and L.-Å. Nyman ${ }^{2}$ \\ 1 Max-Planck-Institut für Radioastronomie, Auf dem Hügel 69, 53121 Bonn, Germany \\ e-mail: schuller@mpifr-bonn.mpg.de \\ 2 European Southern Observatory, Alonso de Cordova 3107, Casilla 19001, Santiago 19, Chile \\ Received 26 April 2006 / Accepted 18 May 2006
}

\section{ABSTRACT}

\begin{abstract}
Context. The M 16 nebula is a relatively nearby HII region, powered by O stars from the open cluster NGC 6611, which borders to a Giant Molecular Cloud. Radiation from these hot stars has sculpted columns of dense obscuring material on a few arcmin scales. The interface between these pillars and the hot ionised medium provides a textbook example of a Photodissociation Region (PDR).

Aims. To constrain the physical conditions of the atomic and molecular material with submillimeter spectroscopic observations.

Methods. We used the APEX submillimeter telescope to map a $\sim 3^{\prime} \times 3^{\prime}$ region in the CO $J=3-2$, 4-3 and 7-6 rotational lines, and a subregion in atomic carbon lines. We also observed $\mathrm{C}^{18} \mathrm{O}(3-2)$ and $\mathrm{CO}(7-6)$ with longer integrations on five peaks found in the $\mathrm{CO}(3-2)$ map. The large scale structure of the pillars is derived from the molecular lines' emission distribution. We estimate the magnitude of the velocity gradient at the tips of the pillars and use LVG modelling to constrain their densities and temperatures. Excitation temperatures and carbon column densities are derived from the atomic carbon lines.

Results. The atomic carbon lines are optically thin and excitation temperatures are of order $60 \mathrm{~K}$ to $100 \mathrm{~K}$, well consistent with observations of other HII region-molecular cloud interfaces. We derive somewhat lower temperatures from the CO line ratios, of order $40 \mathrm{~K}$. The $\mathrm{CI} / \mathrm{CO}$ ratio is around 0.1 at the fingers tips.
\end{abstract}

Key words. HII regions - ISM: individual objects: M 16 - radio lines: ISM - submillimeter

\section{Introduction}

The interplay of massive stars with their surrounding interstellar medium, though of critical importance in the energy budget of a galaxy, is still poorly understood due to observational limitations, and to the generally large distance to high mass star forming regions. Located only $1.8 \pm 0.5 \mathrm{kpc}$ away (Bonatto et al. 2006), the M 16 (Eagle) nebula is one of the best templates for detailed analysis of the environment of high-mass stars. This nebula is associated with the NGC 6611 star cluster, which contains more than two dozen stars of spectral type earlier than B0 (Duchêne et al. 2001) and hundreds of lower mass stars and has an age of $1.3 \pm 0.3 \mathrm{Myr}$ (Bonatto et al. 2006). To the south of this cluster, the radiation from these hot stars has sculpted columns of dense obscuring material on a few arcmin scales, usually referred to as "fingers" or "pillars", of which Hester et al. (1996) presented stunning Hubble Space Telescope (HST) images. The boundary between the HII region and the dense molecular gas provides an archetype of a Photodissociation Region (PDR).

Indications of present-day star formation near the tips of the fingers are seen e.g. at infrared (IR) wavelengths (Thompson et al. 2002). The fingers have been mapped in the $J=1-0$ line of CO and isotopologues by Pound (1998) with the BIMA array. White et al. (1999) used the JCMT $15 \mathrm{~m}$ and OSO $20 \mathrm{~m}$ radiotelescopes to map the same region in various molecular lines and in the submm continuum. They report $\mathrm{CO}(2-1)$ and $\mathrm{CO}(3-2)$ peak brightness temperatures above $40 \mathrm{~K}$ and $60 \mathrm{~K}$, respectively, and a factor 2-3 lower temperature in $\mathrm{CO}(1-0)$.

Here we report on new observations in two submillimeter windows, conducted with the APEX 12 m telescope. We have

1 This publication is based on data acquired with the Atacama Pathfinder Experiment (APEX). APEX is a collaboration between mapped an area covering the three pillars in $\mathrm{CO}$ rotational lines. In addition, we have mapped the fingertips in the ${ }^{3} \mathrm{P}_{1}-{ }^{3} \mathrm{P}_{0}$ and ${ }^{3} \mathrm{P}_{2}-{ }^{3} \mathrm{P}_{1}$ transitions of atomic carbon to probe the PDR. Details on the observations are given in Sect. 2. Results of modelling the dense molecular phase are addressed in Sect. 3, while the properties of the PDR itself are discussed in Sect. 4.

\section{Observations}

We have used the dual colour FLASH heterodyne receiver (Heyminck et al., this volume) to simultaneously observe the $J=$ 4-3 and $J=7-6$ lines of $\mathrm{CO}$, as well as, also simultaneously, the $492 \mathrm{GHz}$ and $809 \mathrm{GHz}$ transitions of atomic carbon. The CO $J=3-2$ line was mapped using the APEX-2a (Risacher et al., this volume) facility receiver. With both instruments, the backend used was the MPIfR Fast Fourier Transform Spectrometer (Klein et al., this volume), which provides 16384 spectral channels over a $1 \mathrm{GHz}$ bandwidth. Calibration was done by observing the sky, hot and cold loads and using an atmospheric model. This provides spectra in antenna temperature unit. Efficiencies have been determined on observations of planets for all receivers (Güsten et al., this volume) and applied to the measured signal. The spectra were then converted to the main beam temperature scale, using beam efficiencies of 0.73 at $345 \mathrm{GHz}, 0.60$ at 460 and $492 \mathrm{GHz}$, and 0.47 at $809 \mathrm{GHz}$. The overall calibration uncertainty of the data is estimated to be $\sim 20 \%$. The pointing was checked every hour on the nearby sources Sgr B2(N) and G10.62-0.38 and was found to be stable within 3". However,

the Max-Planck-Institut für Radioastronomie, the European Southern Observatory, and the Onsala Space Observatory. 
Table 1. Log of the observations. The $T_{\text {sys }}$ column gives typical single side band system temperatures.

\begin{tabular}{|c|c|c|c|}
\hline Transition & $\begin{array}{l}\text { Frequency } \\
{[\mathrm{GHz}]}\end{array}$ & $\begin{array}{l}T_{\text {sys }} \\
{[\mathrm{K}]} \\
\end{array}$ & Date of observation \\
\hline $\mathrm{CO}(3-2)$ & 345.796 & 230 & 17-18 Nov. 2005 \\
\hline $\mathrm{C}^{18} \mathrm{O}(3-2)$ & 329.331 & 330 & 18 Nov. 2005 \\
\hline $\mathrm{CO}(4-3)$ & 461.041 & 800 & $\left\{\begin{array}{l}28 \text { Jul. } 2005+ \\
1 \text { Oct. } 2005+\end{array}\right.$ \\
\hline $\mathrm{CO}(7-6)$ & 806.652 & 2500 & 7-8 Apr. 2006 \\
\hline CI ${ }^{3} \mathrm{P}_{1}-{ }^{3} \mathrm{P}_{0}$ & 492.161 & 850 & 02 Aug. $2005+$ \\
\hline $\mathrm{CI}{ }^{3} \mathrm{P}_{2}-{ }^{3} \mathrm{P}_{1}$ & 809.342 & 2000 & 04-05 Oct. 2005 \\
\hline
\end{tabular}

parts of the observations were done at elevations above $80^{\circ}$, where the tracking becomes somewhat less accurate.

We performed position switched observations, with a fixed reference position to the east of the easternmost finger and at an offset of $120^{\prime \prime}, 0^{\prime \prime}$ relative to our $(0,0)$ position, which is $\alpha_{2000}=18^{\mathrm{h}} 18^{\mathrm{m}} 52^{\mathrm{s}} .19, \delta_{2000}=-13^{\circ} 48^{\prime} 58^{\prime \prime} \cdot 6$. All offsets used in the following are relative to that position. We mapped a $2^{\prime} \times 2^{\prime}$ area with $7^{\prime \prime}$ spacing in both directions in $\mathrm{CO}(4-3)$ and $\mathrm{CO}(7-6)$, and $3^{\prime} \times 3^{\prime}$ with $10^{\prime \prime}$ spacing in $\mathrm{CO}(3-2)$. Based on the peaks found in the $\mathrm{CO}(3-2)$ map, five positions were also observed in the (3-2) transition of $\mathrm{C}^{18} \mathrm{O}$ (see Sect. 3.3 and Table 2). Due to moderate weather conditions for $\mathrm{CO}(7-6)$ in the first observing runs, we repeated the $\mathrm{CO}(4-3)$ and (7-6) observations with longer integrations in April 2006 toward these peak positions. A limited $40^{\prime \prime} \times 20^{\prime \prime}$ region covering the tip of the eastern most finger and a $30^{\prime \prime}$ strip on the tip of the middle finger were mapped in atomic carbon lines, with 7 " spacing in both cases (Sect. 4). The log of the observations is reported in Table 1.

\section{Molecular material}

\subsection{Large scale structure}

A map of the $\mathrm{CO}(3-2)$ emission is shown superimposed on an $8 \mu \mathrm{m}$ Spitzer image in Fig. 1. The $8 \mu \mathrm{m}$ emission mostly comes from small dust grains and polycyclic aromatic hydrocarbons (PAHs) excited by the incoming radiation. It traces the edge of the dusty structure, while the molecular emission peaks somewhat deeper in the columns, as expected from a transition from ionised to atomic to molecular medium. The integrated intensity in the $\mathrm{CO}(4-3)$ line is shown overlaid on the HST data from Hester et al. (1996) in Fig. 2. Given the pointing uncertainties mentioned above, the structure of the $\mathrm{CO}(4-3)$ agrees remarkably well with the columns of dust seen in the HST image.

The 3-dimensional kinematics of the region has been discussed e.g. by Pound (1998). Our data are fully consistent with the picture that they derived. In particular, our data confirm that the $\mathrm{CO}$ emission along the easternmost column arises from two distinct cores. We also observed velocity gradients (see below) with position angles mostly pointing to the nearby $\mathrm{O}$ stars.

\subsection{Dense cores}

On the tip of the eastern most column, we measure a difference in $V_{\mathrm{LSR}}$ of $2.15 \pm 0.07 \mathrm{~km} \mathrm{~s}^{-1}$ between offset positions $\left(+10^{\prime \prime},+20^{\prime \prime}\right)$ and $\left(0,-10^{\prime \prime}\right)$ in the $\mathrm{CO}(3-2)$ line. This corresponds to a velocity gradient of $7.8 \pm 0.3 \mathrm{~km} \mathrm{~s}^{-1} \mathrm{pc}^{-1}$, in good agreement with Pound (1998), who found a value of $7.1 \mathrm{~km} \mathrm{~s}^{-1} \mathrm{pc}^{-1}$ when scaled to the distance of $1.8 \mathrm{kpc}$ used in the present paper.

We derived excitation temperatures by correcting the main beam temperatures for the Rayleigh-Jeans approximation,

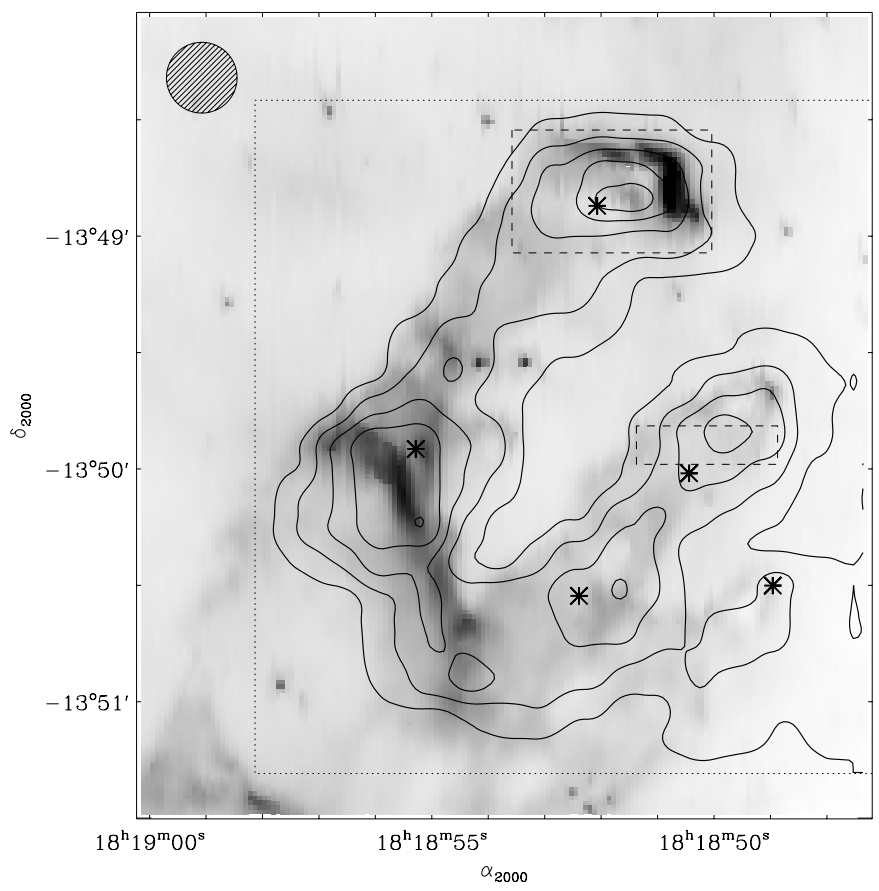

Fig. 1. Contour map of the $\mathrm{CO}(3-2)$ emission, integrated over the +20 to $+30 \mathrm{~km} \mathrm{~s}^{-1} V_{\mathrm{LSR}}$ range, overlaid on a Spitzer $8 \mu \mathrm{m}$ image from the GLIMPSE survey (Benjamin et al. 2003). Contours correspond to 30, $60, \ldots 180 \mathrm{~K} \mathrm{~km} \mathrm{~s}^{-1}$ in integrated $T_{\mathrm{MB}}$. The APEX beam at $345 \mathrm{GHz}$ is shown in the upper left corner. The dotted line shows the limits of our $\mathrm{CO}(3-2)$ data. The asterisks show the positions where we ran LVG modelling (Sect. 3.3) and the frames drawn in dashed lines delineate the areas mapped in atomic carbon lines (Sect. 4).

assuming that the $\mathrm{CO}$ lines are optically thick and that the emission is filling the main beam. At position offset $\left(-10^{\prime \prime}, 0\right)$, the three transitions of $\mathrm{CO}$ give similar temperatures around $54 \pm 7 \mathrm{~K}$, suggesting that the molecular material is close to thermal equilibrium.

\subsection{Gas temperature and column density}

To determine the physical parameters at different positions in the fingers, we used the $\mathrm{CO}$ observations and a spherically symmetric Large Velocity Gradient (LVG) statistical equilibrium code, with the cosmic background as only radiation field. In order to compare the $\mathrm{CO}(7-6)$ and $\mathrm{CO}(4-3)$ observations with the LVG results, we smoothed the $\mathrm{CO}(7-6)$ data to the spatial resolution of the $\mathrm{CO}(4-3)$.

From the $\mathrm{CO}(3-2) / \mathrm{C}^{18} \mathrm{O}(3-2)$ line ratios, we estimated the optical depths of the $\mathrm{CO}(3-2)$ lines, and derived $\mathrm{CO}$ column densities by comparing with LVG simulations. In this calculation, we used ${ }^{16} \mathrm{O} /{ }^{18} \mathrm{O}=407$, as derived from the relation given by Wilson \& Rood (1994) as a function of galactocentric distance, with $d_{\mathrm{GC}}=6.3 \mathrm{kpc}$ (Bonatto et al. 2006). For positon ($\left.10^{\prime \prime}, 0^{\prime \prime}\right)$, the non-detection of $\mathrm{C}^{18} \mathrm{O}(3-2)$ at $v_{\mathrm{LSR}}=23.6 \mathrm{~km} \mathrm{~s}^{-1}$ gives an upper limit to the $\mathrm{CO}$ column density of $10^{17} \mathrm{~cm}^{-2}$. For the other positions, the LVG modelling gives a range of possible column densities, as reported in Table 2; our results agree with the average $\mathrm{CO}$ column density derived by Pound (1998) and with the $\mathrm{C}^{18} \mathrm{O}$ column densities of White et al. (1999).

Using these values for the $\mathrm{CO}$ column densities, we further constrained the gas properties by running LVG calculations for several densities and temperatures. We found that the $\mathrm{CO}(7-6)$ and (4-3) lines are thermalized for densities higher than a few 

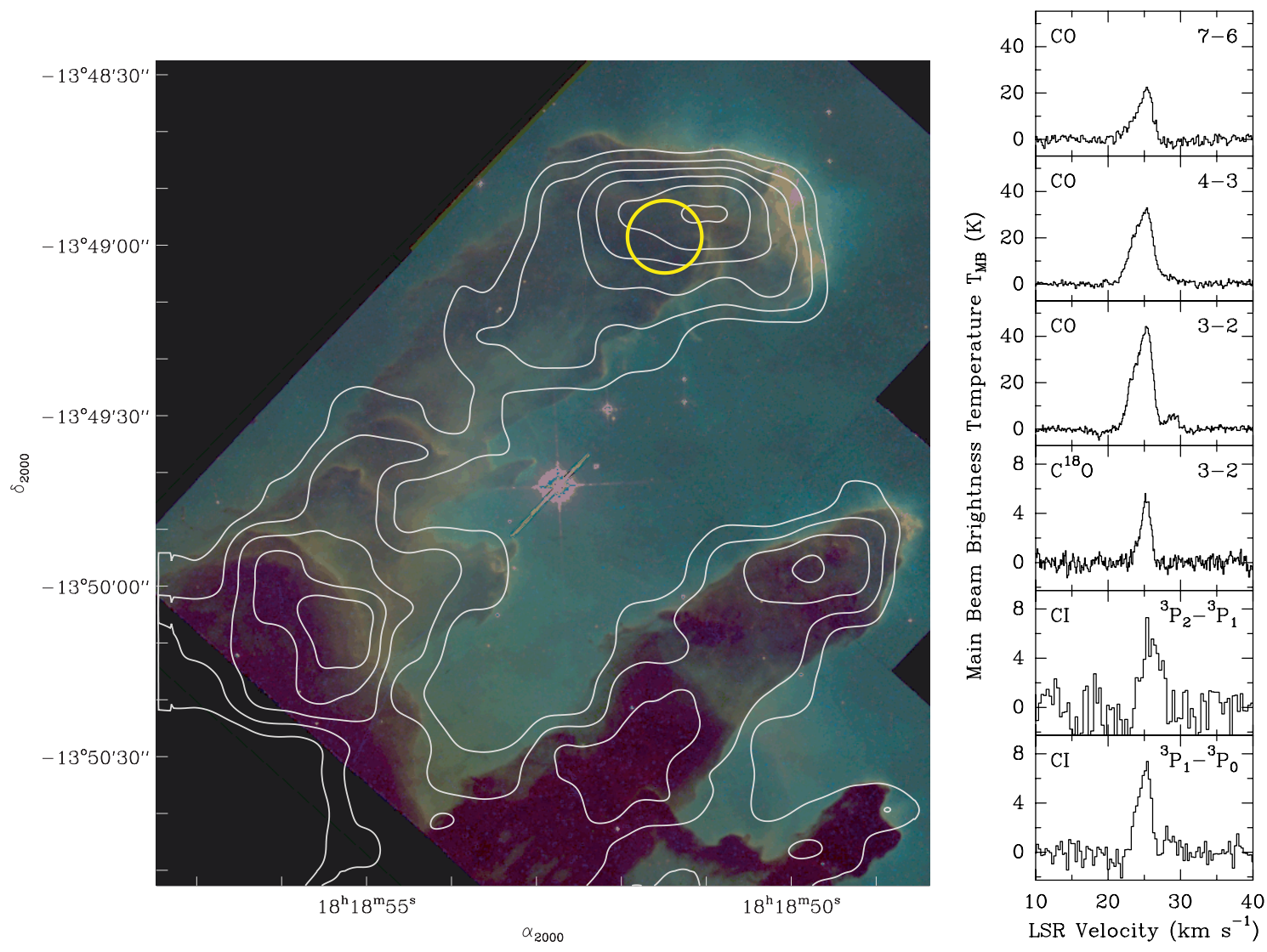

Fig. 2. Left hand side: contour map of the $\mathrm{CO}(4-3)$ emission, integrated over 20-28 $\mathrm{km} \mathrm{s}^{-1}$, overlaid on an HST composite image ([OIII], $\mathrm{H}_{\alpha}$ and [SII] filters) from Hester et al. (1996). Contour levels are shown for $\int T_{\mathrm{MB}} \mathrm{d} v$ from 40 to $165 \mathrm{~K} \mathrm{~km} \mathrm{~s}^{-1}$ in steps of $25 \mathrm{~K} \mathrm{~km} \mathrm{~s}{ }^{-1}$. The circle marks the size of the APEX beam for the CO (4-3) line and is placed at the position toward which the spectra in the right hand side of this figure were taken. Right hand side, top to bottom: spectra of the $\mathrm{CO}(7-6),(4-3),(3-2)$, the $\mathrm{C}^{18} \mathrm{O}(3-2)$ and the $\mathrm{CI}^{3} \mathrm{P}_{2}-{ }^{3} \mathrm{P}_{1}$ and ${ }^{3} \mathrm{P}_{1}-{ }^{3} \mathrm{P}_{0}$ lines taken toward the $-10^{\prime \prime}, 0$ offset position marked in the left part of this figure.

Table 2. Results of the CO lines modelling. Columns 4 and 5 give the gas kinetic temperatures, as derived by comparing the observed $T_{\mathrm{MB}}$ of $\mathrm{CO}(4-3)$ and (7-6), respectively, with the LVG models. Offsets are relative to the $(0,0)$ position given in Sect. 2.

\begin{tabular}{lllll}
\hline \hline $\begin{array}{l}\text { Offset } \\
{\left[{ }^{\prime \prime},{ }^{\prime \prime}\right]}\end{array}$ & $\begin{array}{l}v_{\mathrm{lsr}} \\
{\left[\mathrm{km} \mathrm{s}^{-1}\right]}\end{array}$ & $\begin{array}{l}N(\mathrm{CO}) \\
{\left[\mathrm{cm}^{-2}\right]}\end{array}$ & $\begin{array}{l}T_{\mathrm{CO}(4-3)} \\
{[\mathrm{K}]}\end{array}$ & $\begin{array}{l}T_{\mathrm{CO}(7-6)} \\
{[\mathrm{K}]}\end{array}$ \\
\hline$-10,0$ & 23.6 & $\leq 1 \times 10^{17}$ & $45 \pm 7$ & \\
$-10,0$ & 25.4 & $8 \times 10^{17}-1 \times 10^{19}$ & $54 \pm 8$ & $56 \pm 9$ \\
$+40,-60$ & 24.6 & $8 \times 10^{17}-6 \times 10^{18}$ & $40 \pm 6$ & $42 \pm 6$ \\
$-30,-70$ & 22.4 & $6 \times 10^{17}-1 \times 10^{19}$ & $50 \pm 9$ & $57 \pm 7$ \\
$-50,-100$ & 21.3 & $6 \times 10^{17}-1 \times 10^{19}$ & $30 \pm 4$ & $32 \pm 6$ \\
$0,-100$ & 21.4 & $2 \times 10^{17}-6 \times 10^{18}$ & $51 \pm 9$ & $47 \pm 7$ \\
\hline
\end{tabular}

$10^{4} \mathrm{~cm}^{-3}$. Both lines are optically thick at the column densities that we computed (Table 2); therefore, the observed $T_{\mathrm{MB}}$ give direct measures of the kinetic temperature of the gas. Other authors (Pound 1998; White et al. 1999) reported densities of $3-5 \times 10^{4} \mathrm{~cm}^{-3}$ in the fingers, with higher values $\left(\sim 2 \times 10^{5} \mathrm{~cm}^{-3}\right)$ at the fingertips. Thus, the temperatures reported in Table 2 are derived from LVG models with densities above $10^{4} \mathrm{~cm}^{-3}$. For lower densities, they would correspond to lower limits to the true kinetic temperature of the gas. Our estimates are computed by assuming beam filling factors of 1 . The temperatures we derived do not change significantly over the range of column densities computed for each position and the variation is, in any case, within the error bars, corresponding to a calibration uncertainty of $20 \%$ for each line.

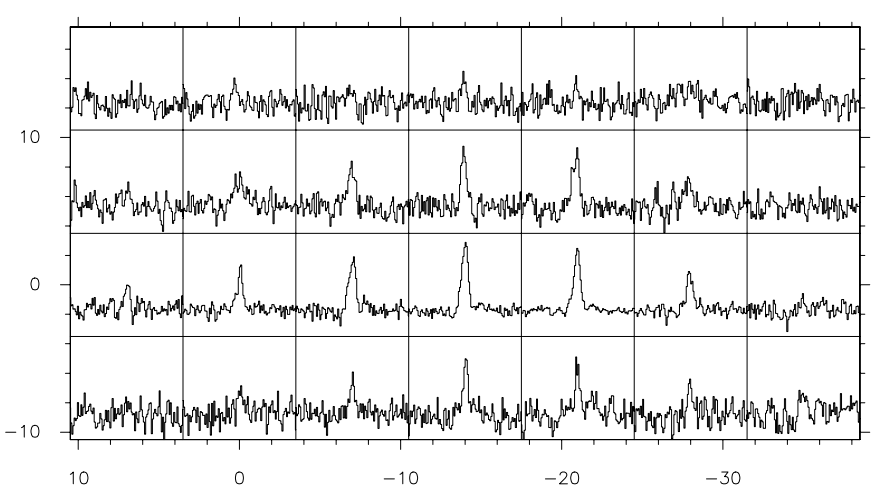

Fig. 3. Map of the atomic carbon line at $492 \mathrm{GHz}$ covering the edge of the northernmost finger. Axes show the offsets (in arcsec) with respect to the $(0,0)$ position (Sect. 2). Each spectrum is shown over the $10-40 \mathrm{~km} \mathrm{~s}^{-1}$ range in $V_{\mathrm{LSR}}$, with a vertical scale going from -5 to $+15 \mathrm{~K}$ in $T_{\mathrm{MB}}$. Excitation temperatures derived from the $492 \mathrm{GHz}$ and $809 \mathrm{GHz}$ transitions are given in Table 3 .

\section{Atomic carbon lines}

In the north-south direction, [CI] $492 \mathrm{GHz}$ extends over at least $\sim 30^{\prime \prime}$, or $0.25 \mathrm{pc}$ at the distance of M 16 (see map in Fig. 3). Using Eqs. (7) and (8) in Zmuidzinas et al. (1988), we derived excitation temperatures and opacities for both [CI] lines, using the measured main beam temperatures for both transitions. This assumes a uniform excitation temperature, and that the levels are 
Table 3. Peak main beam temperatures of the [CI] lines. The excitation temperatures and line opacities are computed after Zmuidzinas et al. (1988), using the $T_{\mathrm{MB}}$ scale for both lines. Typical uncertainties are of order $-20 \mathrm{~K}$ to $+30 \mathrm{~K}$ for $T_{\mathrm{ex}}$, and \pm 0.1 for opacities (see text). Column densities are derived from $T_{\mathrm{ex}}$ and $\tau(1-0)$ after Keene et al. (1985). Offsets are relative to the (0,0) position (Sect. 2).

\begin{tabular}{|c|c|c|c|c|c|c|c|c|c|}
\hline $\begin{array}{l}\mathrm{O} \\
{\left[{ }^{\prime \prime}\right]}\end{array}$ & $\begin{array}{l}\text { set } \\
{["]}\end{array}$ & $\begin{array}{l}\overline{T_{\mathrm{MB}}(1-0)} \\
{[\mathrm{K}]}\end{array}$ & $\begin{array}{l}v_{\mathrm{LSR}} \\
{\left[\mathrm{km} \mathrm{s}^{-1}\right]}\end{array}$ & $\begin{array}{l}T_{\mathrm{MB}}(2-1) \\
{[\mathrm{K}]}\end{array}$ & $\begin{array}{l}v_{\mathrm{LSR}} \\
{\left[\mathrm{km} \mathrm{s}^{-1}\right]}\end{array}$ & $\begin{array}{l}T_{\mathrm{ex}} \\
{[\mathrm{K}]}\end{array}$ & $\overline{\tau \tau(1-0)}$ & $\overline{\tau(2-1)}$ & $\begin{array}{l}N(\mathrm{CI}) \\
{\left[\mathrm{cm}^{-2}\right]}\end{array}$ \\
\hline-21 & 0 & 10.8 & $25.37(0.02)$ & 11.6 & $25.94(0.06)$ & 61 & 0.24 & 0.31 & $3.2 \times 10^{17}$ \\
\hline-14 & 0 & 12.2 & $25.54(0.03)$ & 14.3 & $25.76(0.09)$ & 71 & 0.23 & 0.31 & $3.8 \times 10^{17}$ \\
\hline-7 & 0 & 9.2 & $25.60(0.06)$ & 10.4 & $25.70(0.17)$ & 66 & 0.18 & 0.24 & $2.9 \times 10^{17}$ \\
\hline 0 & 0 & 7.2 & $25.75(0.06)$ & 9.7 & $25.52(0.10)$ & 93 & 0.09 & 0.14 & $1.8 \times 10^{17}$ \\
\hline-35 & -63 & 10.3 & $23.10(0.06)$ & 14.3 & $22.95(0.12)$ & 103 & 0.12 & 0.19 & $2.6 \times 10^{17}$ \\
\hline
\end{tabular}

thermally populated. Since both lines were observed simultaneously, systematic errors should cancel out in this analysis, so that using a $10 \%$ uncertainty for each measured $T_{\mathrm{MB}}$ is reasonable. The resulting uncertainties are of order -20 to $+30 \mathrm{~K}$ for $T_{\mathrm{ex}}$ and \pm 0.1 for opacities.

The results are shown in Table 3 for the central part of the map covering the tip of the first finger, and for offset position $\left(-35^{\prime \prime}, 63^{\prime \prime}\right)$ at the tip of the second finger, where we have good $\mathrm{S} / \mathrm{N}$ spectra in both transitions. We find optically thin lines, with opacities in the range $0.1-0.3$, and excitation temperatures in the range $60-100 \mathrm{~K}$, i.e. in the upper part of the temperature range computed by Zmuidzinas et al. (1988) in various sources. We also derived excitation temperatures from LVG modelling, and found similar results, in the range $80-120 \mathrm{~K}$.

We computed CI column densities using the same formalism as described in the Appendix of Keene et al. (1985). They are reported in the last column of Table 3 . The corresponding $\mathrm{CI} / \mathrm{CO}$ ratios are around 0.1 at the tips of both fingers. However, $\mathrm{CI}$ and $\mathrm{C}^{18} \mathrm{O}$ emissions are likely to trace distinct material, and additional observations are considered to constrain the variations of $\mathrm{CI} / \mathrm{CO}$ in the region.

\section{Summary and conclusion}

We have observed the transitions of atomic carbon for the first time toward the M16 nebula, and derived excitation temperatures for the atomic material around $60-100 \mathrm{~K}$, similar to the values found in other HII region-molecular cloud interfaces. The kinetic temperatures derived from $\mathrm{CO}$ transitions seem somewhat lower, around $40 \mathrm{~K}$ in most positions. This is consistent with the picture where the atomic carbon traces the PDR at the edge of the dense molecular columns. The CI to CO ratio is $\sim 0.1$ at the fingers tips. Additional observations and comparison with PDR models are needed to further constrain the atomic to molecular transition.

Acknowledgements. We are grateful to F. Boone and A. Belloche for their valuable help in various aspects of the data processing.

\section{References}

Benjamin, R. A., Churchwell, E. B., Babler, B., et al. 2003, PASP, 115, 953 Bonatto, C., Santos, J. F. C., \& Bica, E. 2006, A\&A, 445, 567

Duchêne, G., Simon, T., Eislöffel, J., \& Bouvier, J. 2001, A\&A, 379, 147

Hester, J., Scowen, P. A., Sankrit, R., et al. 1996, AJ, 111, 2349

Keene, J., Blake, G. A., Phillips, T. G., Huggins, P. J., \& Beichman, C. A. 1985, ApJ, 299, 967

Pound, M. W. 1998, ApJ, 493, L113

Thompson, R. I., Smith, B. A., \& Hester, J. J. 2002, ApJ, 570, 749

White, G. J., Nelson, R. P., Holland, W. S., et al. 1999, A\&A, 342, 233

Wilson, T. L., \& Rood, R. 1994, ARA\&A, 32, 191

Zmuidzinas, J., Betz, A. L., Boreiko, R. T., \& Goldhaber, D. M. 1988, ApJ, 335, 774 